Article

\title{
Synthesis and Fungicidal Activity of Novel Chloro-Containing 1-Aryl-3-oxypyrazoles with an Oximino Ester or Oximino Amide Moiety
}

\author{
Yuanyuan Liu ${ }^{1, *}$, Yi $\mathrm{Li}^{2}{ }^{2, *}$, Nanqing Chen ${ }^{3}$, Kunzhi $\mathrm{Lv}^{3}$, Chao Zhou ${ }^{1}$, Xiaohui Xiong ${ }^{2}$ and \\ Fangshi Li $^{3}$
}

1 Department of Chemical and Pharmaceutical Engineering, Southeast University ChengXian College, Nanjing 210088, China

2 College of Food Science and Light Industry, Nanjing Tech University, Nanjing 211816, China

3 College of Science, Nanjing Tech University, Nanjing 211816, China

* Authors to whom correspondence should be addressed;

E-Mails: liuyuanyuan1985419@163.com (Y.L.); liynj2012@njtech.edu.cn (Y.L.);

Tel./Fax: +86-25-5866-2849 (Y.L.); +86-25-5813-9432 (Y.L.).

Received: 25 April 2014; in revised form: 9 June 2014 / Accepted: 12 June 2014 /

Published: 17 June 2014

\begin{abstract}
Six novel chloro-containing 1-aryl-3-oxypyrazoles TMa-TMf with an oximino ester or an oximino amide moiety were prepared by the reaction of 1-aryl-1H-pyrazol-3-ols with benzyl bromide. Their structures were characterized by ${ }^{1} \mathrm{H}-\mathrm{NMR},{ }^{13} \mathrm{C}-\mathrm{NMR}, \mathrm{IR}, \mathrm{MS}$, and elemental analysis. A preliminary in vitro bioassay indicated that compounds TMa, TMe and TMf displayed excellent fungicidal activity against Rhizoctonia solani and could be used as potential lead compounds for further development of novel fungicides.
\end{abstract}

Keywords: synthesis; fungicidal activity; chloro-containing 1-aryl-3-oxypyrazoles; structure-activity relationships

\section{Introduction}

Since the discovery of the strobilurin fungicide azoxystrobin by Syngenta scientists [1], this novel fungicide class has occupied an important position in the agrochemical market due to its lower mammalian toxicity, wider spectrum, and higher bioactivity [2-4]. Generally, the chemical structure of strobilurin fungicides can be divided into three parts: (A) an (E)- $\beta$-methyl methoxyiminoacetate or an 
isosteric (E)- $\beta$-methyl methoxyacrylate moiety as pharmacophore; (B) an aromatic bridge moiety; and (C) a side chain. Previous researchers have mainly focused on maintaining the pharmacophore but changing the side chain, and some new strobilurins such as trifloxystrobin, SYP-1620 and pyraclostrobin (Figure 1) have been commercialized [5-8]. Our group has also devoted considerable effort to the development of novel fungicides. Bioactive (alkyl)oxyacetate, thiazolidine-2-thione, $(O$-acetyl)glucopyranosyl or benzoyl moieties were introduced into the 1-aryl-3-oxypyrazole structure of pyraclostrobin to replace its methoxycarbamate pharmacophore which could not be prepared in an environmentally friendly way, and some novel aryloxypyrazoles with good fungicidal activity were reported [9-11].

Figure 1. Design strategy for the target products TM.

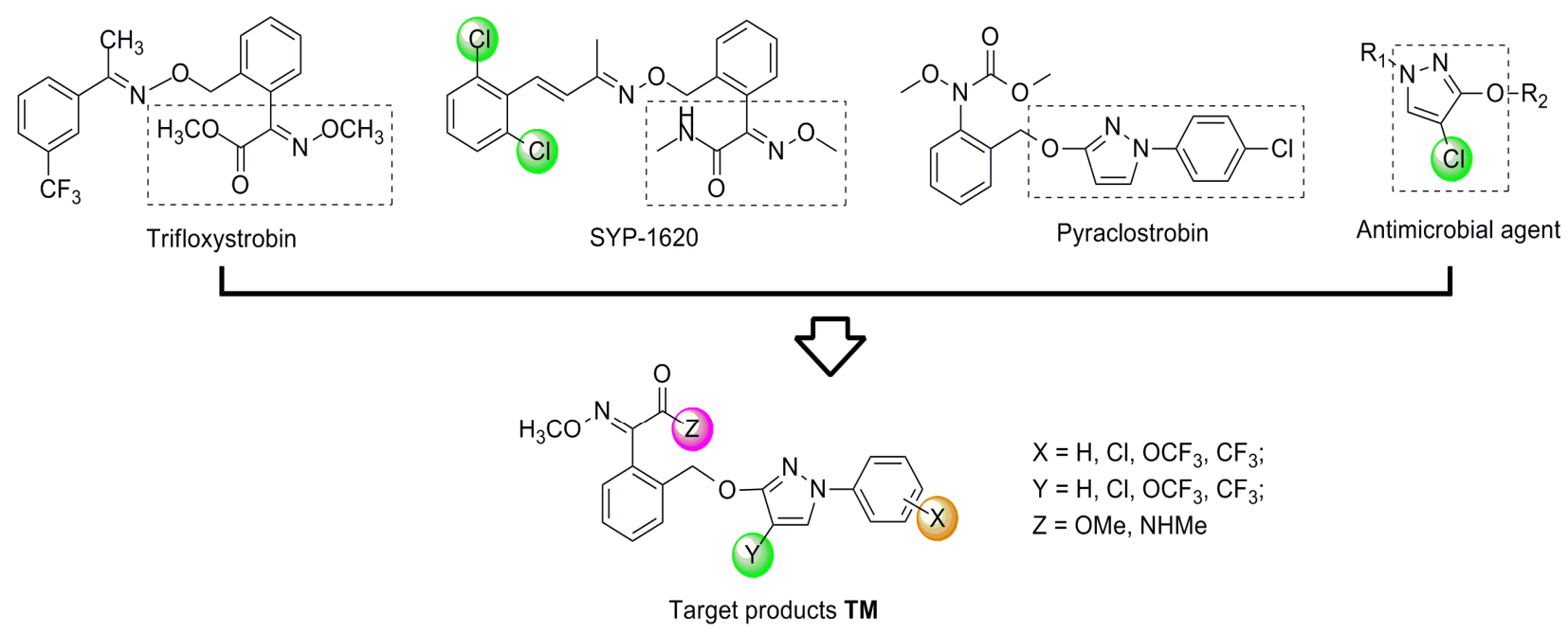

Furthermore, several biological studies have also indicated the 4-chloro-3-oxypyrazole moiety as a bioactive structure, and side chain modifications such as introduction of chlorine can indeed lead to a better fungicidal efficacy [12,13]. In continuation of our studies on fungicidal aryloxypyrazoles, the chlorine and oximino ester or oximino amide pharmacophore of strobilurins were introduced into the 1-aryl-3-oxypyrazole structure of pyraclostrobin according to the principle of active parts combination, and new target products TM were designed (Figure 1).

In this paper, the synthesis of six novel chloro-containing 1-aryl-3-oxypyrazoles TMa-TMf with an oximino ester or an oximino amide moiety is reported. Meanwhile, their fungicidal activity has been investigated with the aim of understanding the structure-activity relationships and developing novel fungicides. Preliminary in vitro bioassay data indicated that some compounds showed excellent fungicidal activity against Rhizoctonia solani, especially TMa, TMe and TMf, which displayed a higher activity than pyraclostrobin at a dosage of $0.1 \mu \mathrm{g} / \mathrm{mL}$.

\section{Results and Discussion}

\subsection{Chemistry}

Intermediate 4-chloro-1-aryl-1H-pyrazol-3-ols IIa-IId were synthesized from arylhydrazines via addition-cyclization, oxidation and chlorination (Scheme 1) $[14,15]$. A previous report by Li and Yang 
described that the benzyl bromide (E)-methyl 2-(2-(bromomethyl)phenyl)-2-(methoxyimino)acetate $\mathbf{V}$ could be prepared from 1-(o-tolyl)ethanone via a serial of reactions including oxidation, esterification, oximation and bromination [16]. However, due to the fact the oxidation was carried out under heterogeneous conditions, the yield was low, so in our procedure, we optimized the reaction conditions, and the oxidation was carried out by phase-transfer catalysis with tetrabutylammonium bromide $\left(\mathrm{Bu}_{4} \mathrm{~N}^{+} \mathrm{Br}^{-}\right)$under alkaline conditions, which gave 2-oxo-2-(o-tolyl)acetic acid III in $80 \%$ yield (Scheme 2). In the following step, the esterification of III was carried out in methanol, using concentrated sulfuric acid as catalyst. The compound obtained was directly used for oximation without further purification. The bromination of oximino ester IV was carried out in a molar ratio of IV to $\mathrm{N}$-bromosuccinimide (NBS) 1:1.1 equiv. in $\mathrm{CCl}_{4}$ as solvent, and with benzoyl peroxide (BPO) as catalyst.

Scheme1. Synthesis of intermediate 4-chloro-1-aryl-1H-pyrazol-3-ols IIa-IId.

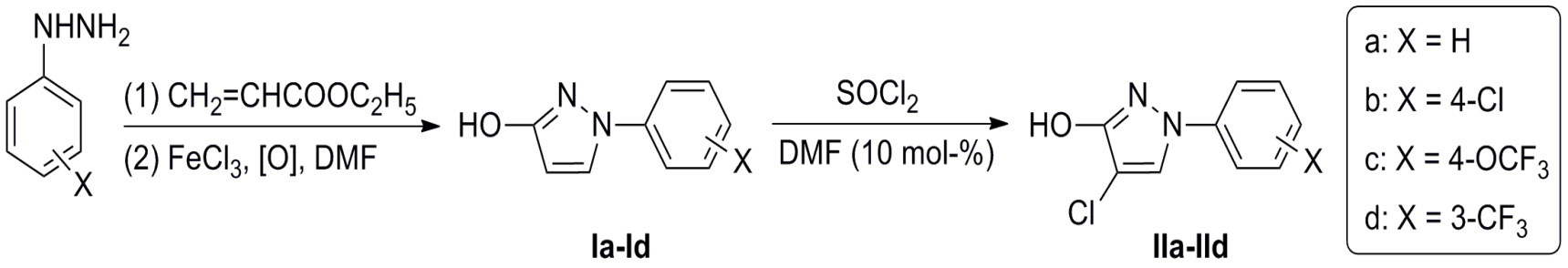

Scheme 2. Synthesis of intermediate benzyl bromide V.

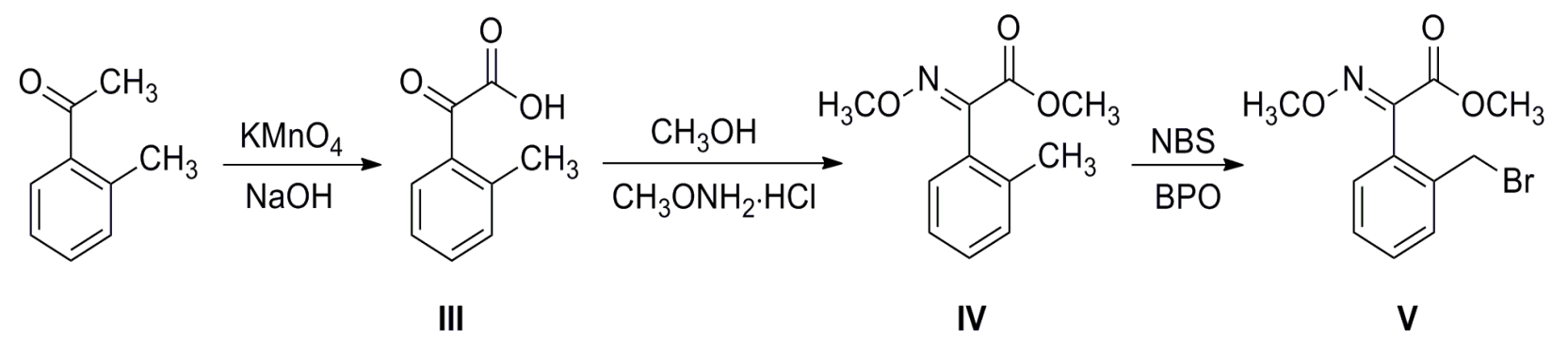

In our previous studies, we have prepared several ethyl 2-(1,5-diaryl-1H-pyrazol-3-yloxy)acetates by the substitution reaction of 1,5-diaryl- $1 H$-pyrazol-3-ols with ethyl 2-bromoacetate in acetone, using potassium carbonate $\left(\mathrm{K}_{2} \mathrm{CO}_{3}\right)$ as acid-binding agent [11]. Motivated by this reaction, in our procedure, hydroxypyrazoles Ib and IIa-IId were allowed to react with benzyl bromide $\mathbf{V}$ in a 1:1.1 molar equiv. ratio in boiling acetone in the presence of $\mathrm{K}_{2} \mathrm{CO}_{3}$, which afforded the target products TMa-TMe in $80 \%-82 \%$ yield as sole isolable products (Scheme 3 ). The reaction process for TMf was carried out in a molar ratio of TMe to methylamine in anhydrous methanol (30\%) 1:3 equiv. at reflux temperature until TMe was completely consumed as judged by TLC. The crude product was purified via column chromatography to give the pure TMf in $90 \%$ isolated yield (Scheme 3).

The structures of TMa-TMf were confirmed by their NMR spectra. In the ${ }^{1} \mathrm{H}-\mathrm{NMR}$ spectra, as a result of the deshielding effect of aryl and chloro groups, the $\mathrm{CH}$ of the pyrazole ring in TMa-TMd appeared at low field $(\delta 7.68-7.81 \mathrm{ppm})$ as a singlet, whereas the corresponding $\mathrm{CH}$ proton in TMe-TMf appeared at $\delta 7.65 \mathrm{ppm}$ and $\delta 5.85 \mathrm{ppm}$, respectively, as two doublets with coupling constants around $2.5 \mathrm{~Hz}$. The aromatic protons of TMa-TMf resonated in the range of $\delta 7.79-7.19 \mathrm{ppm}$ in ${ }^{1} \mathrm{H}-\mathrm{NMR}$ spectra, and the ${ }^{13} \mathrm{C}-\mathrm{NMR}$ signals were observed around $\delta 146.6-114.3 \mathrm{ppm}$. The chemical 
shifts of MeO H-atoms in TMa-TMe appeared as two singlets around $\delta 4.06 \mathrm{ppm}$ and $\delta 3.84 \mathrm{ppm}$, respectively, whereas one $\mathrm{MeO}$ proton signal in TMf was absent and replaced by the Me proton at $\delta$ $2.89 \mathrm{ppm}$. All compounds exhibited a carbonyl $(\mathrm{C}=\mathrm{O}){ }^{13} \mathrm{C}$ signal at the lowest field in the region of $\delta 163.9-163.3$ ppm.

Scheme 3. Synthesis of target products TMa-TMf.

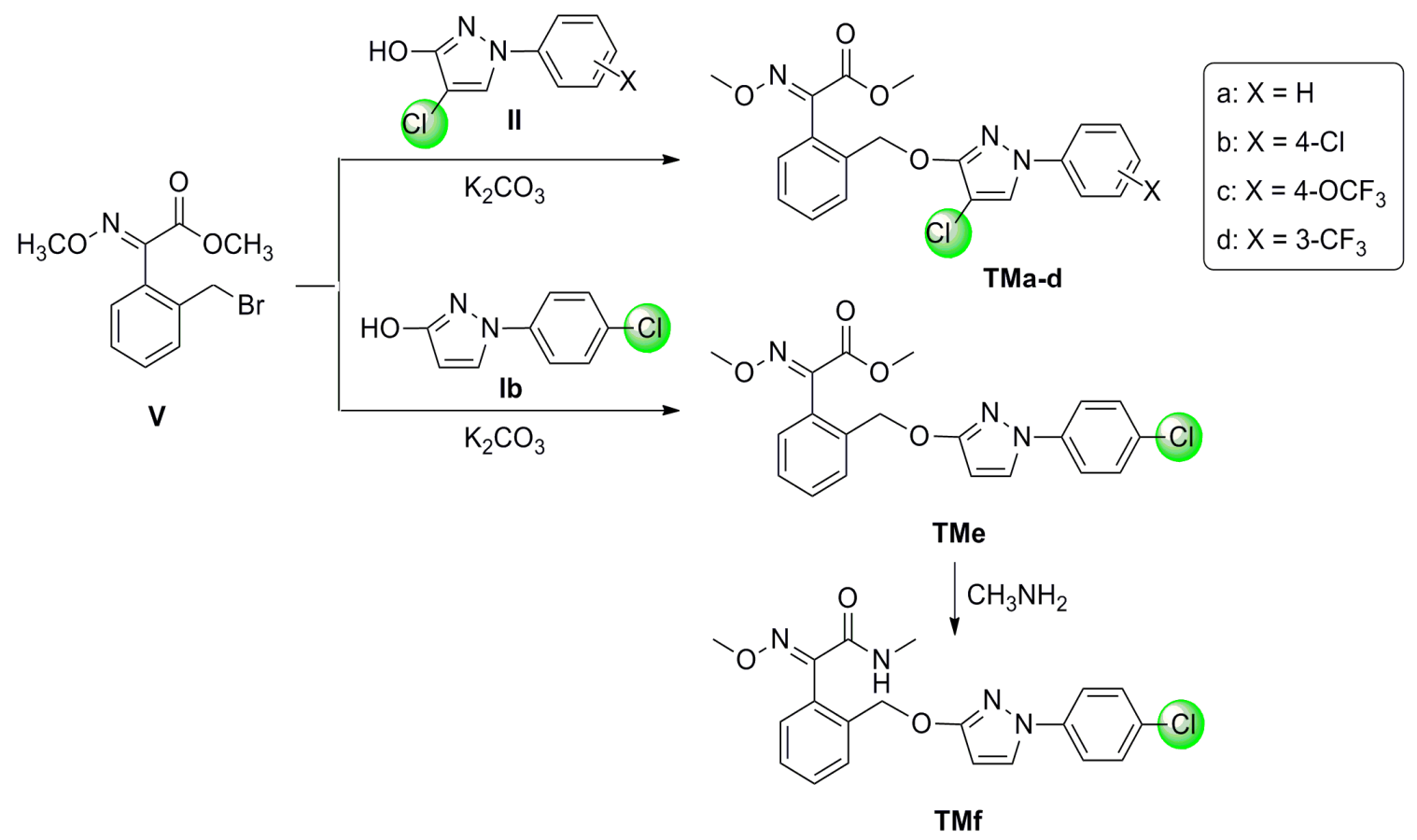

\subsection{Fungicidal Activity}

Products TMa-TMf were screened for fungicidal activity against Rhizoctonia solani at the dosages of 10,1 , and $0.1 \mu \mathrm{g} / \mathrm{mL}$, respectively. As can be seen in Tables 1 and 2, most compounds have good fungicidal activity at $10 \mu \mathrm{g} / \mathrm{mL}$, especially TMa, TMe and TMf, with $100 \%$ antifungal activity. When the concentration was reduced to $0.1 \mu \mathrm{g} / \mathrm{mL}$, TMe, TMa and TMf also had $99 \%, 83 \%$ and $71 \%$ inhibition rates, which were better than those of the reference compound pyraclostrobin. Compound TMe had an optimal $\mathrm{EC}_{90}$ lower than $0.1 \mu \mathrm{g} / \mathrm{mL}$, which was better than TMa $\left(\mathrm{EC}_{90}=0.20 \mu \mathrm{g} / \mathrm{mL}\right)$ and TMf $\left(\mathrm{EC}_{90}=0.32 \mu \mathrm{g} / \mathrm{mL}\right)$. The 4-chloropyrazole containing a $\mathrm{Cl}$ on the phenyl ring (TMb) displayed higher activity than that with an electron-donating $\mathrm{CF}_{3} \mathrm{O}$ (TMc) or electron-withdrawing $\mathrm{CF}_{3}$ (TMd) group. According to the different positions of chlorine, the sequence of fungicidal activity is $\mathrm{Cl}$-substituted phenyl ring $>\mathrm{Cl}$-substituted pyrazole ring. For example, the structure only containing a $\mathrm{Cl}$ on the phenyl ring (TMe) showed better fungicidal activity than that on the pyrazole ring (TMa). However, with increasing number of the $\mathrm{Cl}$ groups, the fungicidal activity was decreased, as seen in the comparison of TMa and TMe vs. TMb, which might because the larger molecular volume was unfavourable for the intracellular uptake and transport in the fungus. In terms of the pharmacophore, the compound with an oximino ester moiety (TMe) had better fungicidal activity than that with an oximino amide (TMf) or a methoxycarbamate moiety (pyraclostrobin). The present work indicated that TMa, TMe and TMf could be used as potential lead compounds for further studies of novel fungicides. 
Table 1. Antifungal activity of tested compounds ( $\%$ inhibition).

\begin{tabular}{|c|c|c|c|c|}
\hline \multirow{2}{*}{ Compounds } & \multirow{2}{*}{ Structure } & \multicolumn{3}{|c|}{ Rhizoctonia solani $^{\text {a }}$} \\
\hline & & $10 \mu \mathrm{g} / \mathrm{mL}$ & $1 \mu \mathrm{g} / \mathrm{mL}$ & $0.1 \mu \mathrm{g} / \mathrm{mL}$ \\
\hline ТМа & & +++ & +++ & +++ \\
\hline TMb & & +++ & +++ & + \\
\hline TMe & & +++ & +++ & +++ \\
\hline TMf & & +++ & +++ & ++ \\
\hline TMc & & ++ & + & + \\
\hline TMd & & ++ & +++ & ++ \\
\hline Pyraclostrobin & & ++ & + & + \\
\hline
\end{tabular}

${ }^{a}$ Activity is expressed in four categories: $(-)<20 \%,(+) 21 \%-50 \%,(++) 51 \%-80 \%$, and (+++) $81 \%-100 \%$.

Table 2. Antifungal activity and $\mathrm{EC}_{90}$ values of compounds TMa, TMe and TMf.

\begin{tabular}{ccccc}
\hline \multirow{2}{*}{ Compounds } & \multicolumn{3}{c}{ concn $(\boldsymbol{\mu g} / \mathbf{m L})^{\mathbf{a}}$} & \multirow{2}{*}{$\mathbf{E C}_{\mathbf{9 0}}\left(\boldsymbol{\mu} \mathbf{g} \mathbf{~ m L}^{-\mathbf{1}}\right)$} \\
\cline { 2 - 4 } & $\mathbf{1 0}$ & $\mathbf{1}$ & $\mathbf{0 . 1}$ & 0.20 \\
TMa & 100.00 & 98.23 & 83.40 & $<0.1$ \\
TMe & 100.00 & 100.00 & 98.94 & 0.32 \\
TMf & 100.00 & 94.70 & 71.40 & \\
\hline
\end{tabular}

${ }^{\mathrm{a}} 100.00=$ total kill.

\section{Experimental}

\subsection{General Information}

Melting points were measured on an X-4 microscope electrothermal apparatus (Taike, Beijing, China) and were uncorrected. NMR spectra were obtained in $\mathrm{CDCl}_{3}$ or DMSO-d $\mathrm{d}_{6}$ on a Brucker NMR spectrometer operating at $400 \mathrm{MHz}$ for ${ }^{1} \mathrm{H}$ and $100 \mathrm{MHz}$ for ${ }^{13} \mathrm{C}$, with TMS as an internal standard. Elemental analyses were performed on a Flash EA-1112 elemental analyzer. 


\subsection{Synthesis and Characterization}

Intermediates 1-aryl-1H-pyrazol-3-ols Ia-Id were prepared according to the reported method, and the spectral data matched those previously reported [14].

\subsubsection{General Procedure for the Synthesis of IIa-IId}

A mixture of $\mathrm{SOCl}_{2}(5 \mathrm{~mL}), \mathbf{I a}-\mathbf{I d}(1.0 \mathrm{mmol})$, and a catalytic amount of DMF $(0.1 \mathrm{mmol})$ were heated under reflux for about $4 \mathrm{~h}$ (monitored by TLC), then excess $\mathrm{SOCl}_{2}$ was evaporated and $\mathrm{H}_{2} \mathrm{O}$ ( $200 \mathrm{~mL}$ ) was added with good stirring. The precipitate that formed was filtered off, washed with $\mathrm{H}_{2} \mathrm{O}$, and then purified by a silica-gel column chromatography (petroleum ether/EtOAc $=5: 1$ ) to afford IIa-IId.

4-Chloro-1-phenyl-1H-pyrazol-3-ol (IIa). Yield 80\%; White solid; mp 183-184 ${ }^{\circ} \mathrm{C}$; ${ }^{1} \mathrm{H}-\mathrm{NMR}$ (DMSO-d ${ }_{6}$ ) $\delta 11.00$ (s, 1H, OH), 8.54 (s, 1H, CH), 7.69-7.21 (m, 5H, Ar-H). Anal. calcd for $\mathrm{C}_{9} \mathrm{H}_{7} \mathrm{ClN}_{2} \mathrm{O}: \mathrm{C} 55.54$, H 3.63, N 14.39; found C 55.65, H 3.62, N 14.34.

4-Chloro-1-(4-chlorophenyl)-1H-pyrazol-3-ol (IIb). Yield 85\%; White solid; mp 204-205 ${ }^{\circ} \mathrm{C}$; ${ }^{1} \mathrm{H}-\mathrm{NMR}\left(\mathrm{CDCl}_{3}\right) \delta 7.69(\mathrm{~s}, 1 \mathrm{H}, \mathrm{CH}), 7.46-7.39$ (m, 4H, Ar-H). Anal. calcd for $\mathrm{C}_{9} \mathrm{H}_{6} \mathrm{Cl}_{2} \mathrm{~N}_{2} \mathrm{O}: \mathrm{C} 47.19$, H 2.64, N 12.23; found C 47.12, H 2.64, N 12.26.

4-Chloro-1-(4-(trifluoromethoxy)phenyl)-1H-pyrazol-3-ol (IIc). Yield 78\%; White solid; mp 172-173 ${ }^{\circ} \mathrm{C}$; ${ }^{1} \mathrm{H}-\mathrm{NMR}\left(\mathrm{CDCl}_{3}\right) \delta 7.62(\mathrm{~s}, 1 \mathrm{H}, \mathrm{CH}), 7.40(\mathrm{~d}, J=8.8 \mathrm{~Hz}, 2 \mathrm{H}, \mathrm{Ar}-\mathrm{H}), 7.25(\mathrm{~d}$, $J=8.8 \mathrm{~Hz}, 2 \mathrm{H}, \mathrm{Ar}-\mathrm{H})$. Anal. calcd for $\mathrm{C}_{10} \mathrm{H}_{6} \mathrm{ClF}_{3} \mathrm{~N}_{2} \mathrm{O}_{2}: \mathrm{C} 43.11, \mathrm{H} \mathrm{2.17}, \mathrm{N} 10.05$; found $\mathrm{C} 43.33, \mathrm{H}$ 2.13, N 10.12 .

4-Chloro-1-(3-(trifluoromethyl)phenyl)-1H-pyrazol-3-ol (IId).Yield 76\%; White solid; mp 210-211 ${ }^{\circ} \mathrm{C}$; ${ }^{1} \mathrm{H}-\mathrm{NMR}\left(\mathrm{CDCl}_{3}\right) \delta 7.74(\mathrm{~s}, 1 \mathrm{H}, \mathrm{CH}), 7.66-7.46(\mathrm{~m}, 4 \mathrm{H}, \mathrm{Ar}-\mathrm{H})$. Anal. calcd for $\mathrm{C}_{10} \mathrm{H}_{6} \mathrm{ClF}_{3} \mathrm{~N}_{2} \mathrm{O}: \mathrm{C}$ 45.73, H 2.30, N 10.67; found C 45.55, H 2.26, N 10.75.

\subsubsection{General Procedure for the Synthesis of III}

A mixture of 1-(o-tolyl)ethanone (10 g, $74.6 \mathrm{mmol}), \mathrm{H}_{2} \mathrm{O}(150 \mathrm{~mL}), \mathrm{NaOH}(2 \mathrm{~g}, 50 \mathrm{mmol})$ and $\mathrm{Bu}_{4} \mathrm{~N}^{+} \mathrm{Br}^{-}(0.1 \mathrm{~g}, 0.295 \mathrm{mmol})$ was stirred at $0{ }^{\circ} \mathrm{C}$, then $\mathrm{KMnO}_{4}(23 \mathrm{~g}, 0.146 \mathrm{~mol})$ was added gradually. The mixture was stirred at $30{ }^{\circ} \mathrm{C}$ until the color of the solution remained unchanged. The black precipitate was filtered off, and the filtrate was neutralized by $\mathrm{NaHSO}_{3}$ solution, acidified with $\mathrm{HCl}$ (36.5\%) and filtered. The filtrate was extracted with EtOAc, and then the solvent was removed under reduced pressure to afford 2-oxo-2-(o-tolyl)acetic acid (III). Yield 80\%; White oil; ${ }^{1} \mathrm{H}-\mathrm{NMR}\left(\mathrm{CDCl}_{3}\right) \delta$ 7.83-7.25 (m, 4H, Ar-H), $2.52\left(\mathrm{~s}, 3 \mathrm{H}, \mathrm{CH}_{3}\right)$. Anal. calcd for $\mathrm{C}_{9} \mathrm{H}_{8} \mathrm{O}_{3}$ : C 65.85, $\mathrm{H}$ 4.91; found C 65.62, H 4.93 .

\subsubsection{General Procedure for the Synthesis of IV}

A mixture of III $(5 \mathrm{~g}, 0.03 \mathrm{~mol}), \mathrm{H}_{2} \mathrm{SO}_{4}(1 \mathrm{~mL})$, and $\mathrm{MeOH}(100 \mathrm{~mL})$ was heated to reflux until the starting material had been completely consumed as judged by TLC. The mixture was neutralized by $\mathrm{NaHCO}_{3}$, and $\mathrm{CH}_{3} \mathrm{ONH}_{2} \cdot \mathrm{HCl}(3 \mathrm{~g}, 0.036 \mathrm{~mol})$ was added. The reaction was monitored by TLC, then 
excess $\mathrm{MeOH}$ was removed under reduced pressure. The residue was neutralized by $\mathrm{NaHCO}_{3}$, extracted by EtOAc, dried, filtered, and evaporated under reduced pressure. It was then purified by a silica-gel column chromatography (petroleum ether/EtOAc $=8: 1)$ to afford $(E)$-methyl 2-(methoxyimino)-2-(o-tolyl)acetate (IV). Yield 70\%; White solid; mp 63-64 ${ }^{\circ} \mathrm{C} ;{ }^{1} \mathrm{H}-\mathrm{NMR}\left(\mathrm{CDCl}_{3}\right) \delta 7.31-7.10$ (m, $4 \mathrm{H}, \mathrm{Ar}-\mathrm{H}), 4.05\left(\mathrm{~s}, 3 \mathrm{H}, \mathrm{OCH}_{3}\right), 3.87\left(\mathrm{~s}, 3 \mathrm{H}, \mathrm{OCH}_{3}\right), 2.19\left(\mathrm{~s}, 3 \mathrm{H}, \mathrm{CH}_{3}\right)$. Anal. calcd for $\mathrm{C}_{11} \mathrm{H}_{13} \mathrm{NO}_{3}$ : C 63.76, H 6.32, N 6.76; found C 63.98, H 6.29, N 6.74.

\subsubsection{General Procedure for the Synthesis of $\mathbf{V}$}

To the solution of IV $(5 \mathrm{~g}, 24.0 \mathrm{mmol})$ in $\mathrm{CCl}_{4}(150 \mathrm{~mL})$ was added NBS $(4.7 \mathrm{~g}, 26.4 \mathrm{mmol})$ and BPO (1 g, $4.13 \mathrm{mmol})$. The reaction mixture was heated to reflux until the starting material had been completely consumed as judged by TLC. The precipitate was filtered off, and the solvent was evaporated under reduced pressure. The residue was purified by a silica-gel column chromatography (petroleum ether/EtOAc $=15: 1)$ to give $(E)$-methyl 2-(2-(bromomethyl)phenyl)-2-(methoxyimino)acetate (V). Yield 85\%; Yellow oil; ${ }^{1} \mathrm{H}-\mathrm{NMR}\left(\mathrm{CDCl}_{3}\right) \delta$ 7.47-7.13 (m, 4H, Ar-H), $4.32\left(\mathrm{~s}, 2 \mathrm{H}, \mathrm{CH}_{2}\right)$, $4.04\left(\mathrm{~s}, 3 \mathrm{H}, \mathrm{OCH}_{3}\right), 3.85\left(\mathrm{~s}, 3 \mathrm{H}, \mathrm{OCH}_{3}\right)$. Anal. calcd for $\mathrm{C}_{11} \mathrm{H}_{12} \mathrm{BrNO}_{3}: \mathrm{C} 46.18, \mathrm{H} 4.23, \mathrm{~N} 4.90$; found C 46.32, H 4.21, N 4.88 .

\subsubsection{General Procedure for the Synthesis of TMa-TMe}

To a solution of $\mathbf{I b}$ and $\mathbf{I I a}-\mathbf{I I d}(1.0 \mathrm{mmol})$ in acetone $(30 \mathrm{~mL})$ was added $\mathrm{K}_{2} \mathrm{CO}_{3}(1.5 \mathrm{mmol})$. The mixture was refluxed for $15 \mathrm{~min}$ and $\mathbf{V}(1.05 \mathrm{mmol})$ was added slowly. The mixture was refluxed for about $4 \mathrm{~h}$, then $\mathrm{K}_{2} \mathrm{CO}_{3}$ was filtered off. The solvent was evaporated under reduced pressure. The residue was purified by a silica-gel column chromatography (petroleum ether/EtOAc $=10: 1$ ) to afford products TMa-TMe.

(E)-Methyl 2-(2-\{[(4-chloro-1-phenyl-1H-pyrazol-3-yl)oxy]methyl $\}$ phenyl)-2-(methoxyimino)acetate (TMa). Yield 80\%; White solid; mp 104-105 ${ }^{\circ} \mathrm{C} ;{ }^{1} \mathrm{H}-\mathrm{NMR}\left(\mathrm{CDCl}_{3}\right) \delta 7.73(\mathrm{~s}, 1 \mathrm{H}, \mathrm{CH}), 7.63(\mathrm{~d}$, $J=7.4 \mathrm{~Hz}, 1 \mathrm{H}$, oximino-ArH), 7.53-7.19 (m, 8H, $\mathrm{ArH}), 5.25$ (s, 2H, $\left.\mathrm{CH}_{2}\right), 4.04$ (s, 3H, MeO), 3.84 (s, $3 \mathrm{H}, \mathrm{MeO}) .{ }^{13} \mathrm{C}-\mathrm{NMR}\left(\mathrm{CDCl}_{3}\right) \delta 163.3,158.9,149.4,139.7,134.8,130.1,129.5,129.4,128.9,128.5$, 128.1, 125.7, 125.6, 117.6, 98.3, 69.5, 63.9, 53.1. IR (KBr): 3147, 2949, 2895, 1734, 1598, 1557, 1511, 1463, 1438, 1406, 1356, 1323, 1223, 1122, 1063, 1012, 984, 947, 895, 862, 795, 756, 684. MS (ESI): calcd for $[\mathrm{M}+\mathrm{H}]^{+}$400.11, $[\mathrm{M}+\mathrm{Na}]^{+}$422.09; found $[\mathrm{M}+\mathrm{H}]^{+} 400.11,[\mathrm{M}+\mathrm{Na}]^{+}$422.09. Anal. calcd for $\mathrm{C}_{20} \mathrm{H}_{18} \mathrm{ClN}_{3} \mathrm{O}_{4}$ : C 60.08, H 4.54, N 10.51; found C 59.83, H 4.58, N 10.57.

(E)-Methyl 2-(2-\{[(4-chloro-1-(4-chlorophenyl)-1H-pyrazol-3-yl)oxy]methyl\}phenyl)-2-(methoxyimino) acetate (TMb). Yield 82\%; White solid; mp 147-148 ${ }^{\circ} \mathrm{C} ;{ }^{1} \mathrm{H}-\mathrm{NMR}\left(\mathrm{CDCl}_{3}\right) \delta 7.68(\mathrm{~s}, 1 \mathrm{H}, \mathrm{CH}), 7.61$ (d, $J=7.4 \mathrm{~Hz}, 1 \mathrm{H}$, oximino-ArH), 7.44 (d, $J=8.0 \mathrm{~Hz}, 2 \mathrm{H}, \mathrm{N}-\mathrm{ArH}), 7.40$ (d, $J=8.0 \mathrm{~Hz}, 2 \mathrm{H}, \mathrm{N}-\mathrm{ArH})$, 7.33 (m, 2H, oximino-ArH), 7.21 (dd, $J=1.6,7.4 \mathrm{~Hz}, 1 \mathrm{H}$, oximino-ArH), 5.23 (s, 2H, $\left.\mathrm{CH}_{2}\right), 4.03$ (s, $3 \mathrm{H}, \mathrm{MeO}), 3.84(\mathrm{~s}, 3 \mathrm{H}, \mathrm{MeO}) .{ }^{13} \mathrm{C}-\mathrm{NMR}\left(\mathrm{CDCl}_{3}\right) \delta 163.3,159.0,149.4,138.2,134.7,131.0,130.1$, 129.5, 128.9, 128.5, 128.2, 125.6, 118.6, 98.8, 69.5, 63.9, 53.1. IR (KBr): 3140, 2940, 1721, 1594, $1547,1495,1437,1355,1327,1253,1217,1114,1063,1016,959,829,785,723,689$. MS (ESI): 
calcd for $[\mathrm{M}+\mathrm{H}]^{+}$434.07, $[\mathrm{M}+\mathrm{Na}]^{+}$456.05; found $[\mathrm{M}+\mathrm{H}]^{+}$434.07, $[\mathrm{M}+\mathrm{Na}]^{+}$456.05. Anal. calcd for $\mathrm{C}_{20} \mathrm{H}_{17} \mathrm{Cl}_{2} \mathrm{~N}_{3} \mathrm{O}_{4}$ : C 55.31, H 3.95, N 9.68; found C 55.52, H 3.91, N 9.62.

(E)-Methyl 2-(2-\{[(4-chloro-1-(4-(trifluoromethoxy)phenyl)-1H-pyrazol-3-yl)oxy]methyl\}phenyl)-2(methoxyimino)acetate (TMc). Yield 75\%; White solid; mp $149-150{ }^{\circ} \mathrm{C} ;{ }^{1} \mathrm{H}-\mathrm{NMR}\left(\mathrm{CDCl}_{3}\right) \delta 7.71$ (s, 1H, CH), 7.62 (d, $J=7.2 \mathrm{~Hz}, 1 \mathrm{H}$, oximino-ArH), 7.52 (d, $J=8.4 \mathrm{~Hz}, 2 \mathrm{H}, \mathrm{N}-\mathrm{ArH}), 7.42$ (m, 2H, oximino-ArH), 7.25 (d, $J=8.4 \mathrm{~Hz}, 2 \mathrm{H}, \mathrm{N}-\mathrm{ArH}), 7.21$ (dd, $J=1.6,7.4 \mathrm{~Hz}, 1 \mathrm{H}$, oximino-ArH), 5.24 (s, $\left.2 \mathrm{H}, \mathrm{CH}_{2}\right), 4.04$ (s, 3H, MeO), 3.84 (s, 3H, $\left.\mathrm{MeO}\right) .{ }^{13} \mathrm{C}-\mathrm{NMR}\left(\mathrm{CDCl}_{3}\right) \delta 163.3,159.2,149.4,146.6$, 138.2 , 134.7, 130.1, 129.5, 128.9, 128.5, 128.2, 125.7, 122.2, 118.6, 99.0, 69.6, 63.9, 53.0. IR (KBr): 3144, 2946, 1722, 1607, 1558, 1507, 1441, 1393, 1370, 1298, 1261, 1208, 1146, 1115, 1063, 1017, 957, 842, 808, 759, 728, 685. MS (ESI): calcd for $[\mathrm{M}+\mathrm{H}]^{+} 484.09,[\mathrm{M}+\mathrm{Na}]^{+}$506.07; found $[\mathrm{M}+\mathrm{H}]^{+}$ 484.09, $[\mathrm{M}+\mathrm{Na}]^{+}$506.06. Anal. calcd for $\mathrm{C}_{21} \mathrm{H}_{17} \mathrm{ClF}_{3} \mathrm{~N}_{3} \mathrm{O}_{5}$ : C 52.13, H 3.54, $\mathrm{N} 8.68$; found C 52.35, H 3.60, N 8.61.

(E)-Methyl 2-(2-\{[(4-chloro-1-(3-(trifluoromethyl)phenyl)-1H-pyrazol-3-yl)oxy]methyl phenyl)-2(methoxyimino)acetate (TMd). Yield 83\%; White solid; mp 119-120 ${ }^{\circ} \mathrm{C} ;{ }^{1} \mathrm{H}-\mathrm{NMR}\left(\mathrm{CDCl}_{3}\right) \delta 7.81$ (s, $1 \mathrm{H}, \mathrm{CH}), 7.79(\mathrm{~s}, 1 \mathrm{H}, \mathrm{N}-\mathrm{ArH}), 7.68(\mathrm{~d}, J=8.0 \mathrm{~Hz}, 1 \mathrm{H}, \mathrm{N}-\mathrm{ArH}), 7.62(\mathrm{~d}, J=7.4 \mathrm{~Hz}, 1 \mathrm{H}$, oximino-ArH), 7.51 (t, $J=7.6 \mathrm{~Hz}, 1 \mathrm{H}, \mathrm{N}-\mathrm{ArH}), 7.42$ (m, 3H, oximino-ArH), 7.21 (t, $J=7.6 \mathrm{~Hz}, 1 \mathrm{H}$, $\mathrm{N}-\mathrm{ArH}), 5.26\left(\mathrm{~s}, 2 \mathrm{H}, \mathrm{CH}_{2}\right), 4.05(\mathrm{~s}, 3 \mathrm{H}, \mathrm{MeO}), 3.85(\mathrm{~s}, 3 \mathrm{H}, \mathrm{MeO}) .{ }^{13} \mathrm{C}-\mathrm{NMR}\left(\mathrm{CDCl}_{3}\right) \delta 163.3,159.3$, $149.4,140.0,134.5,130.2$, 130.1, 129.5, 129.0, 128.5, 128.2, 125.6, 122.1, 122.0, 120.1, 114.3, 99.6, 69.7, 63.9, 53.1. IR (KBr): 3140, 2946, 1730, 1598, 1562, 1518, 1458, 1406, 1358, 1328, 1306, 1226, 1169, 1122, 1067, 1014, 948, 889, 794, 758, 692. MS (ESI): calcd for $[\mathrm{M}+\mathrm{H}]^{+} 468.09,[\mathrm{M}+\mathrm{Na}]^{+}$ 490.08; found $[\mathrm{M}+\mathrm{H}]^{+}$468.10, $[\mathrm{M}+\mathrm{Na}]^{+}$490.08. Anal. calcd for $\mathrm{C}_{21} \mathrm{H}_{17} \mathrm{ClF}_{3} \mathrm{~N}_{3} \mathrm{O}_{4}$ : C 53.91, $\mathrm{H} \mathrm{3.66,} \mathrm{N}$ 8.98; found C 54.12, H 3.60, N 8.92.

(E)-Methyl 2-(2-(((1-(4-chlorophenyl)-1H-pyrazol-3-yl)oxy)methyl)phenyl)-2-(methoxyimino)acetate (TMe). Yield 82\%; White solid; mp $131-132{ }^{\circ} \mathrm{C} ;{ }^{1} \mathrm{H}-\mathrm{NMR}\left(\mathrm{CDCl}_{3}\right) \delta 7.66(\mathrm{~d}, J=2.4 \mathrm{~Hz}, 1 \mathrm{H}, \mathrm{CH})$, $7.60(\mathrm{~d}, J=7.2 \mathrm{~Hz}, 1 \mathrm{H}$, oximino-ArH), 7.52 (d, $J=9.0 \mathrm{~Hz}, 2 \mathrm{H}, \mathrm{N}-\mathrm{ArH}), 7.42$ (m, 2H, oximino-ArH), 7.36 (d, $J=9.0 \mathrm{~Hz}, 2 \mathrm{H}, \mathrm{N}-\mathrm{ArH}), 7.20$ (dd, $J=1.8,7.2 \mathrm{~Hz}, 1 \mathrm{H}$, oximino-ArH), 5.85 (d, $J=2.4 \mathrm{~Hz}, 1 \mathrm{H}$, $\mathrm{CH}), 5.18\left(\mathrm{~s}, 2 \mathrm{H}, \mathrm{CH}_{2}\right), 4.08\left(\mathrm{~s}, 3 \mathrm{H}, \mathrm{OCH}_{3}\right), 3.84\left(\mathrm{~s}, 3 \mathrm{H}, \mathrm{OCH}_{3}\right) .{ }^{13} \mathrm{C}-\mathrm{NMR}\left(\mathrm{CDCl}_{3}\right) \delta 163.7,163.0$, 149.1, 138.3, 134.8, 130.1, 129.4, 129.1, 129.0, 128.1, 127.5, 127.3, 119.2, 118.5, 94.2, 68.7, 63.4, 52.6. IR (KBr): 3142, 2997, 2945, 1740, 1589, 1542, 1475, 1357, 1306, 1225, 1069, 1016, 978, 935, 895, 845, 784, 749. MS (ESI): calcd for $[\mathrm{M}+\mathrm{H}]^{+}$400.11, $[\mathrm{M}+\mathrm{Na}]^{+} 422.09$; found $[\mathrm{M}+\mathrm{H}]^{+} 400.10,[\mathrm{M}+\mathrm{Na}]^{+}$ 422.09. Anal. calcd for $\mathrm{C}_{20} \mathrm{H}_{18} \mathrm{ClN}_{3} \mathrm{O}_{4}$ : C 60.08, $\mathrm{H} 4.54, \mathrm{~N} 10.51$; found $\mathrm{C} 59.85, \mathrm{H} 4.59, \mathrm{~N} 10.58$.

\subsubsection{General Procedure for the Synthesis of TMf}

Compound TMe $(0.4 \mathrm{~g}, 1.0 \mathrm{mmol})$ was dissolved in dried $\mathrm{MeOH}(40 \mathrm{~mL})$, and then $\mathrm{CH}_{3} \mathrm{NH}_{2}$ in EtOH $(0.31 \mathrm{~g}, 30 \%)$ was added slowly. The mixture was heated to reflux until the starting material had been completely consumed as judged by TLC analysis. The solvent was evaporated under reduced pressure, and the residue was purified by a silica-gel column chromatography (petroleum ether/EtOAc $=3: 1$ ) to afford TMf. 
(E)-2-(2-(((1-(4-Chlorophenyl)-1H-pyrazol-3-yl)oxy)methyl)phenyl)-2-(methoxyimino)-N-methylacetami de (TMf). Yield 90\%; Yellow oil; ${ }^{1} \mathrm{H}-\mathrm{NMR}\left(\mathrm{CDCl}_{3}\right) \delta 7.65(\mathrm{~d}, J=2.7 \mathrm{~Hz}, 1 \mathrm{H}, \mathrm{CH}), 7.58(\mathrm{~d}, J=6.9 \mathrm{~Hz}$, 1H, oximino-ArH), 7.51 (d, $J=9.0 \mathrm{~Hz}, 2 \mathrm{H}, \mathrm{N}-\mathrm{ArH}), 7.43$ (m, 2H, oximino-ArH), 7.37 (d, $J=9.0 \mathrm{~Hz}$, $2 \mathrm{H}, \mathrm{N}-\mathrm{ArH}), 7.22(\mathrm{dd}, J=1.8,6.9 \mathrm{~Hz}, 1 \mathrm{H}$, oximino-ArH), $6.74(\mathrm{~s}, 1 \mathrm{H}, \mathrm{NH}), 5.85(\mathrm{~d}$, $J=2.7 \mathrm{~Hz}, 1 \mathrm{H}, \mathrm{CH}), 5.17\left(\mathrm{~s}, 2 \mathrm{H}, \mathrm{CH}_{2}\right), 3.95\left(\mathrm{~s}, 3 \mathrm{H}, \mathrm{OCH}_{3}\right), 2.89\left(\mathrm{~d}, J=4.8 \mathrm{~Hz}, 3 \mathrm{H}, \mathrm{CH}_{3}\right) .{ }^{13} \mathrm{C}-\mathrm{NMR}$ $\left(\mathrm{CDCl}_{3}\right) \delta 163.9,162.6,150.8,138.3,135.0,130.1,129.0,128.9,128.3,128.0,127.4,127.3,127.2$, 118.5, 94.2, 68.9, 62.9, 25.9. IR (KBr): IR (KBr): 3406, 3147, 2937, 1682, 1595, 1545, 1484, 1456, $1417,1358,1301,1267,1237,1166,1094,1027,981,932,884,830,783,751$. MS (ESI): calcd for $[\mathrm{M}+\mathrm{H}]^{+}$399.12, $[\mathrm{M}+\mathrm{Na}]^{+}$421.10; found $[\mathrm{M}+\mathrm{H}]^{+}$399.12, $[\mathrm{M}+\mathrm{Na}]^{+}$421.11. Anal. calcd for $\mathrm{C}_{20} \mathrm{H}_{19} \mathrm{ClN}_{4} \mathrm{O}_{3}$ : C 60.23, H 4.80, N 14.05; found C 60.02, H 4.75, N 14.13.

\subsection{Fungicidal Activity Assays}

Fungicidal activity of TMa-TMf against Rhizoctonia solani was evaluated according to the literature procedures $[10,17]$. Compounds TMa-TMf were dissolved in acetone at a concentration of $10 \mathrm{ppm}, 1 \mathrm{ppm}$ and $0.1 \mathrm{ppm}$, respectively, and then added to a sterile agarized Czapek-Dox medium at $45{ }^{\circ} \mathrm{C}$. The control sample contained only one equivalent of acetone. The media were poured onto 8-cm Petri dishes (10 $\mathrm{mL}$ for each dish) and after 2 days inoculated with 5-mm PDA discs of overgrown mycelium. The medium was inoculated by a prick of laboratory needle containing fungus spores. The Petri dishes were incubated at r.t. in the dark. After 4 days, the diameters of the inoculation of the cultures were measured. The percentage inhibition of fungal growth was determined by comparison between the development of fungi colonies on media containing compounds and on the control. Three replicates of each test were carried out. Comparative studies involving pyraclostrobin were carried out under the same conditions using solutions in acetone. For the most active compounds, concentrations that would give $90 \%$ growth inhibition $\left(\mathrm{EC}_{90}\right)$ were calculated by the statistical method of linear regression between the activity and the logarithm of the concentration.

\section{Conclusions}

In summary, six novel chloro-containing 1-aryl-3-oxypyrazoles TMa-TMf with an oximino ester or an oximino amide moiety were synthesized by the reaction of 1-aryl-1H-pyrazol-3-ols with benzyl bromide. Their fungicidal activity were tested in vitro against Rhizoctonia solani, and most compounds displayed good fungicidal activity, especially TMa, with an optimal $\mathrm{EC}_{90}$ lower than $0.1 \mu \mathrm{g} / \mathrm{mL}$, TMe and TMf with $\mathrm{EC}_{90} \mathrm{~S}$ of 0.20 and $0.32 \mu \mathrm{g} / \mathrm{mL}$, respectively, which were better than those of pyraclostrobin. In terms of the different positions of chlorine, the sequence of fungicidal activity is Cl-substituted phenyl $>$ Cl-substituted pyrazole. The fungicidal activity was however decreased with increasing chlorine substitution. The present work indicated that TMa, TMe and TMf could be used as potential lead compounds for further studies of novel fungicides.

\section{Acknowledgments}

The authors gratefully acknowledge the financial supports of the Science Foundation for Youth Scholars of Jiangsu Province (BK20130749) and the Youth Foundation of Southeast University ChengXian College (7303600001) of China. 


\section{Author Contributions}

LY, LY, CN, LK, ZC, XX and LF designed research; LY, LY, CN, LK, ZC, XX and LF performed research and analyzed the data; LY, LY, XX and LF wrote the paper. All authors read and approved the final manuscript.

\section{Conflicts of Interest}

The authors declare no conflict of interest.

\section{References}

1. Clough, J.M.; Godfrey, C.R.A.; de Fraine, P.J. Fungicidal Aryloximinoacetates. EP Patent 472300A2, 26 February 1992.

2. Zhao, P.-L.; Liu, C.-L.; Huang, W.; Wang, Y.-Z.; Yang, G.-F. Synthesis and fungicidal evaluation of novel chalcone-based strobilurin analogues. J. Agric. Food Chem. 2007, 55, 5697-5700.

3. Tu, S.; Xu, L.H.; Ye, L.Y.; Wang, X.; Sha, Y.; Xiao, Z.Y. Synthesis and fungicidal activities of novel indene-substituted oxime ether strobilurins. J. Agric. Food Chem. 2008, 56, 5247-5253.

4. Hwang, I.; Kim, J.; Kim, H.; Kyung, S. Synthesis and SAR of methoxyiminoacetate and methoxyiminoacetamide derivatives as strobilurin analogues. Bull. Korean Chem. Soc. 2009, 30, 1475-1480.

5. Herms, S.; Seehaus, K.; Koehle, H.; Conrath, U. A strobilurin fungicide enhances the resistance of tobacco against tobacco mosaic virus and Pseudomonas syringae pv tabaci. Plant Physiol. 2002, $130,120-127$.

6. Mercader, J.V.; Suarez-Pantaleon, C.; Agullo, C.; Abad-Somovilla, A.; Abad-Fuentes, A. Production and characterization of monoclonal antibodies specific to the strobilurin pesticide pyraclostrobin. J. Agric. Food Chem. 2008, 56, 7682-7690.

7. Wolf, B.; Benoit, R.; Sauter, H.; Grammenos, W.; Wingert, H.; Kuekenhoehner, T.; Hepp, M. Preparation of E-methoximes of 2-(phenoxymethyl)phenylglyoxylates. DE Patent 4042272A1, 2 July 1992.

8. Clough, J.M.; Godfrey, C.R.A.; Streeting, I.T.; Cheetham, R.; de Fraine, P.J.; Bartholomew, D.; Eshelby, J.J. Preparation of [(Pyrimidinyloxy)phenyl]methoxypropenoates and Related Compounds as Agrochemical Fungicides. EP Patent 468695A1, 29 January 1992.

9. Liu, Y.; He, G.; Chen, K.; Li, Y.; Zhu, H. Synthesis, crystal structure, and fungicidal activity of novel 1,5-diaryl-1H-pyrazol-3-oxy derivatives containing oxyacetic acid or oxy(2-thioxothiazolidin3-yl)ethanone moieties. J. Heterocycl. Chem. 2012, 49, 1370-1375.

10. Liu, Y.-Y.; Shi, H.; He, G.-K.; Song, G.-L.; Zhu, H.-J. Synthesis, crystal structures, and fungicidal activity of novel 1,5-diaryl-3-(glucopyranosyloxy)-1H-pyrazoles. Helv. Chim. Acta 2012, 95, 1645-1656.

11. Liu, Y.; Shi, H.; Li, Y.; Zhu, H. Synthesis, crystal structure, and fungicidal activity of novel 1,5-diaryl-1H-pyrazol-3-oxyacetate derivatives. J. Heterocycl. Chem. 2010, 47, 897-902. 
12. Mulla, M.S.; Darwazeh, H.A. Field and Laboratory Investigations on the Control of Susceptible and Resistant Pasture Mosquitoes. In Proceedings and Papers of the Thirty-seventh Annual Conference of the California Mosquito Control Association, Las Angeles, CA, USA, 27-29 January 1969; CMCA Press: Los Angeles, CA, USA, 1970; Volume 37, pp. 76-81.

13. Stierli, D.; Daina, A.; Walter, H.; Tobler, H.; Rajan, R. Novel Microbiocides. WO Patent 2009012998A1, 29 June 2009.

14. Li, Y.; Liu, R.; Yan, Z.; Zhang, X.; Zhu, H. Synthesis, crystal structure and fungicidal activities of new type oxazolidinone-based strobilurin analogues. Bull. Korean Chem. Soc. 2010, 31, 3341-3347.

15. Liu, Y.; He, G.; Chen, K.; Jin, Y.; Li, Y.; Zhu, H. DMF-Catalyzed direct and regioselective C-H functionalization: Electrophilic/nucleophilic 4-halogenation of 3-oxypyrazoles. Eur. J. Org. Chem. 2011, 2011, 5323-5330.

16. Li, G.; Yang, H. Synthesis and antifungal bioactivity of methyl 2-methoxyimino-2-\{2-[(substituted benzylidene)aminooxymethyl]phenyl $\}$ acetate and 2-methoxy imino-2-\{2-[(substituted benzylidene)aminooxymethyl]phenyl $\}-\mathrm{N}-$ methylacetamide derivatives. Chin. J. Chem. 2009, 27, 2055-2060.

17. Wilamowski, J.; Kulig, E.; Sepiol, J.J.; Burgiel, Z.J. Synthesis and in vitro antifungal activity of 1-amino-3,4-dialkylnaphthalene-2-carbonitriles and their analogues. Pest Manag. Sci. 2001, 57, 625-632.

Sample Availability: Samples of the intermediates and target products are available from the authors.

(C) 2014 by the authors; licensee MDPI, Basel, Switzerland. This article is an open access article distributed under the terms and conditions of the Creative Commons Attribution license (http://creativecommons.org/licenses/by/3.0/). 